\title{
Synthesis of a Novel PTH1-34 Analog with Increased Human Serum Albumin Affinity
}

\author{
Si-Da Ruan ${ }^{1} \quad$ Yuan-Zhen Dong ${ }^{1} \quad$ Jian-Guang Lu ${ }^{1} \quad$ Meng-Jia Zhao ${ }^{1}$ Wei-Gen Lu ${ }^{1}$ Jun Feng ${ }^{1 *}$
}

${ }^{1}$ China State Institute of Pharmaceutical Industry, Shanghai, People's
Republic of China

Pharmaceut Fronts 2021;3:e23-e29.
Address for correspondence Wei-Gen Lu, PhD, China State Institute of Pharmaceutical Industry, 285 Gebaini Road, Shanghai 201203, People's Republic of China (e-mail: sipiluwg@163.com).

Jun Feng, PhD, China State Institute of Pharmaceutical Industry, 285 Gebaini Road, Shanghai 201203, People's Republic of China (e-mail: fengj31@aliyun.com).

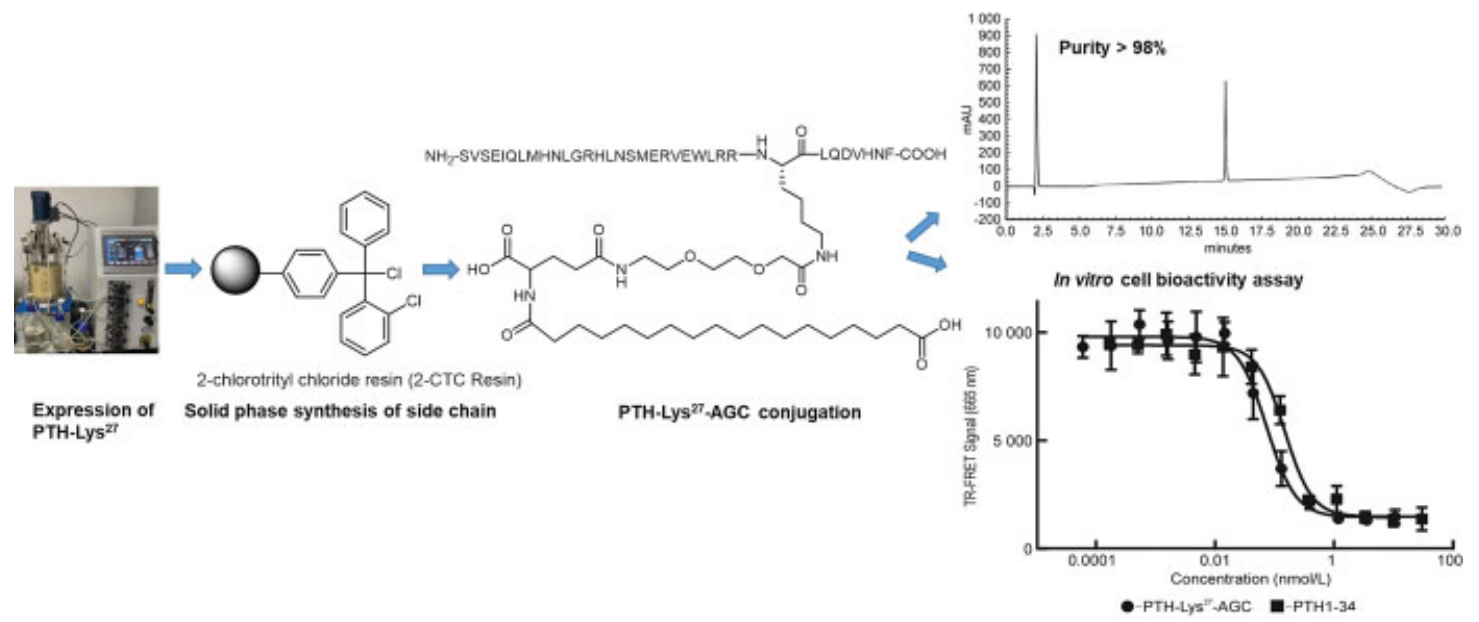

\begin{abstract}
Keywords

- PTH1-34 analog

- fatty acid

- long-acting

- synthesis route
\end{abstract}

Parathyroid hormone (PTH)1-34 is an effective peptide drug for osteoporosis therapy. However, the half-life of PTH1-34 in vivo is short, leading to the need for frequent injections of this drug during its treatment. To prolong the half-life of PTH1-34, a novel PTH1-34 analog was generated based on fatty acid generation, and its synthesis process included recombinant protein expression, side-chain modification, and peptide decoration. The PTH1-34 variant was expressed in Escherichia coli, with a single Lys (position 27) retained as a modification site. The side chain, -AEEA-yGlu-C18 diacid, was synthesized using 2-chlorotrityl chloride resin as a solid support, and then was conjugated to the PTH1-34 variant to form PTH-Lys ${ }^{27}$-AGC. Reversed-phase chromatography confirmed a high final purity ( $>98 \%$ ) of the target compound; in vitro bioactivity tests showed that PTH-1 receptor potency of PTH-Lys ${ }^{27}$-AGC was comparable to that of the native PTH1-34. A competitive human serum albumin binding test demonstrated a high albumin affinity of PTH-Lys ${ }^{27}$-AGC in comparison to PTH1-34. In summary, we developed a novel PTH1-34 analog, PTH-Lys ${ }^{27}$-AGC, which may be a longacting agent for osteoporosis treatment in the future. received

April 2, 2021

accepted

May 28, 2021
DOI https://doi.org/

$10.1055 / \mathrm{s}-0041-1731299$.

ISSN 2628-5088. (c) 2021. The Author(s).

This is an open access article published by Thieme under the terms of the Creative Commons Attribution License, permitting unrestricted use, distribution, and reproduction so long as the original work is properly cited. (https://creativecommons.org/licenses/by/4.0/)

Georg Thieme Verlag KG, Rüdigerstraße 14, 70469 Stuttgart,

Germany 


\section{Introduction}

Osteoporosis often occurs in the elderly, and is a systemic bone metabolism disease characterized by low bone mass and destruction of the bone microstructure, leading to increased bone fragility and tendency of fracture. ${ }^{1}$ It is well known that endogenous parathyroid hormone (PTH), an 84-residue polypeptide synthesized, stored, and secreted by parathyroid epithelial cells, plays an essential role in regulating bone metabolism. ${ }^{2}$ PTH1-34 is a 34 amino acid residue fragment at the $\mathrm{N}$-terminus of $\mathrm{PTH}$, and has been approved by U.S. Food and Drug Administration for the treatment of osteoporosis, yet has a short half-life and requires frequent injection during its administration. Thus, development of long-acting PTH1-34 analog(s) remains urgently needed.

Currently, strategies based on fatty acid (FA) modification is a feasible approach to extending the half-life of peptide drugs because of the high binding affinity of human serum albumin (HSA) for FAs, and the reversible noncovalent interactions between them. ${ }^{3}$ HSA has a long half-life (up to 19 days) in vivo and mediates the in vivo circulation of various endogenous and exogenous ligands, such as FA. ${ }^{4}$ Thus, conjugation of FA, a serum albumin ligand, to therapeutic peptides may prolong the in vivo half-life of those drugs. ${ }^{5}$ For example, the half-life of several peptide drugs, including glucagon-like peptide-1 (GLP-1), growth hormone, calcitonin, and insulin, has been greatly increased via the conjugation of FAs to the corresponding therapeutic peptides. ${ }^{6}$ Several therapeutic biodrugs, such as semaglutide and liraglutide, have been successfully developed via the conjugation of FAs to therapeutic peptides and all conjugates display improved efficiencies in vivo in clinical applications. ${ }^{7}$ However, whether conjugation of a FA to PTH1-34 would extend the half-life of the peptide drug remains unknown.

Methods for the synthesis of peptides commonly focus on two approaches: recombinant protein expression and solidphase chemical synthesis. Chemical synthesis of peptides is often associated with a fast synthesis rate with no risk of contamination by host residue(s), yet with limited usefulness, due to high costs for the generation of functionalgroup-protected amino acids, and environmental pollution caused by the use of large amounts of organic solvents. ${ }^{8}$ In comparison, recombinant expression technology has many advantages, such as low costs, high yield, and environmental friendliness, thus has been widely used for preparation of peptides, particularly those with a length of $>25$ amino acids. In this report, the complete synthetic route for a PTH1-34 analog with high HSA affinity has been described for the first time. Recombinant protein expression was used for the production of the PTH-Lys ${ }^{27}$ sequence (PTH1-34 variant retaining a single Lys as the modification site, PTHLys $^{27}$; - Fig. 1). The peptide backbone was decorated with a

PTH1-34: $\mathrm{NH}_{2}$-SVSEIQLMHNLGKHLNSMERVEWLRKKLQDVHNF-COOH

PTH-Lys ${ }^{27}: \mathrm{NH}_{\underline{2}}$-SVSEIQLMHNLGRHLNSMERVEWLRRKLQDVHNF-COOH

Fig. 1 Sequences of PTH1-34 and PTH-Lys ${ }^{27}$. side chain containing FA and hydrophilic linker to generate a novel compound, PTH-Lys ${ }^{27}$-AGC, which maintained similar in vitro biological potency on target cells with strong albumin binding capacity to the native PTH1-34 along. PTHLys $^{27}$-AGC may therefore represent a long-term peptide drug for the future treatment of osteoporosis.

\section{Results}

\section{Successful Preparation and Identification of PTH-Lys ${ }^{27}$} PTH1-34 variant (PTH-Lys ${ }^{27}$ ) with only one lysine modification site was constructed in Escherichia coli. Inclusion bodies were digested with acid and restriction enzyme to remove fusion tag, and then purified to obtain PTH-Lys ${ }^{27}$. As shown in - Fig. 2A, PTH-Lys ${ }^{27}$ was $95.2 \%$ in its purity, with the molecular weight of 4,173.72 Da, consistent with theoretical calculation (-Fig. 2B). The accurate molecular mass and the sequence of peptide segment were determined by liquid chromatography-mass spectrometry (LC-MS) peptide mapping (-Fig. 2C). The restriction fragments and corresponding molecular weights are shown in -Table 1. Our results
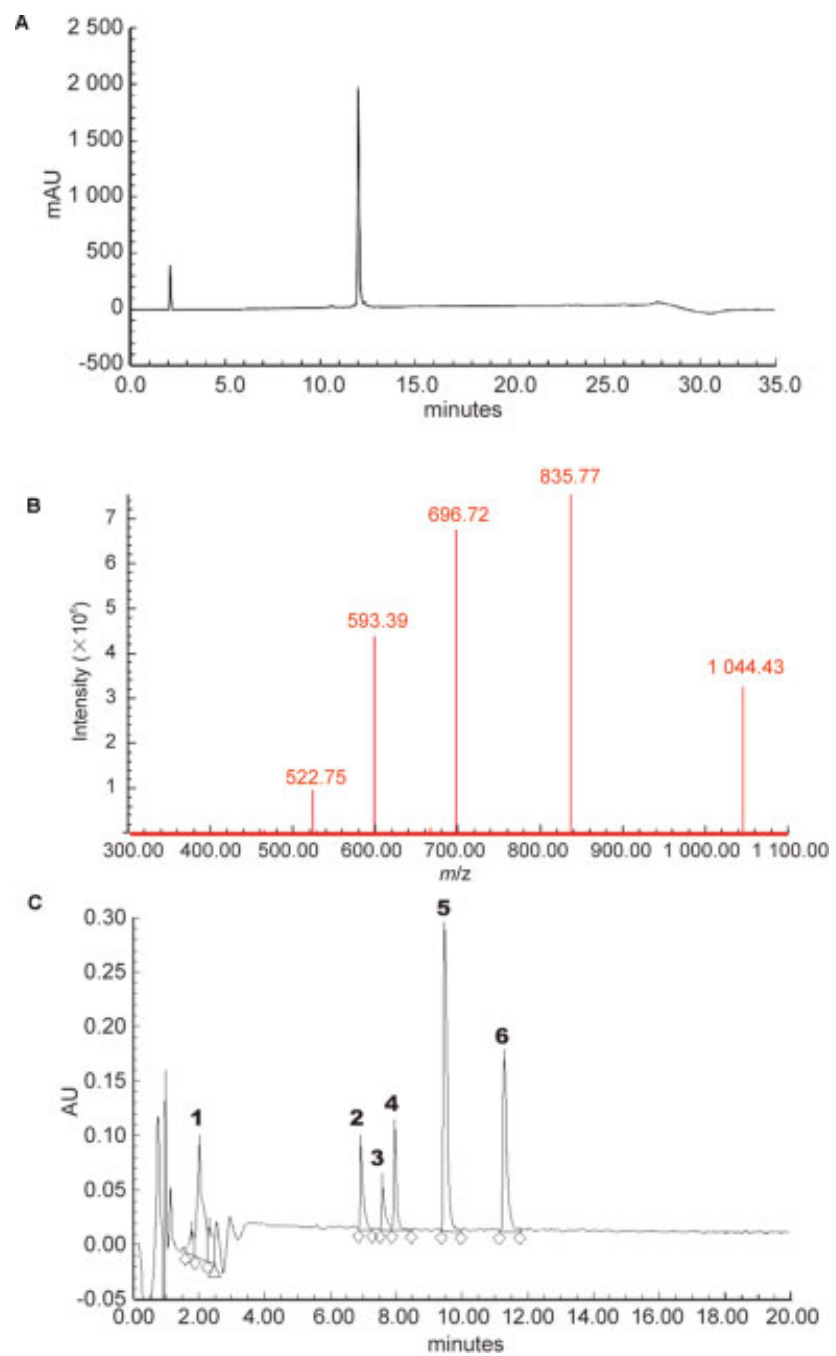

Fig. 2 Characterization of PTH-Lys ${ }^{27}$. (A) HPLC curve, (B) mass spectrum, and (C) peptide map. HPLC, high-performance liquid chromatography. 
Table 1 Peptide map analysis of fragments and sequences of PTH-Lys ${ }^{27}$

\begin{tabular}{|l|l|l|}
\hline No. & $\mathrm{m} / \mathrm{z}$ & Peptide segment \\
\hline 1 & 886.42 & $14-20$ \\
\hline 2 & $1,000.53$ & $27-34$ \\
\hline 3 & 858.48 & $21-26$ \\
\hline 4 & 872.44 & $28-34$ \\
\hline 5 & 702.38 & $21-25$ \\
\hline 6 & $1,484.22$ & $1-13$ \\
\hline
\end{tabular}

showed that all fragments of PTH-Lys ${ }^{27}$ can be identified in peptide mapping, validating the precise product sequence.

\section{Synthesis of NHS-AEEA-yGlu-C18 Ester}

The side chain was assembled by standard Fmoc chemistry as shown in - Fig. 3. Briefly, compound $\mathbf{5}$ was cleaved from resin with a molecular weight of $701.13[\mathrm{M}+\mathrm{H}]^{+}$. To obtain an active terminal carboxyl group of the side chain, $N$-hydroxysuccinimide (HOSu) was added to form NHS ester (compound 6 ). The $M_{\mathrm{w}}$ of the compound 6 was $798.02[\mathrm{M}+\mathrm{H}]^{+}$. Tert-butyl protection was finally removed to prepare NHSAEEA-rGlu-C18 ester (compound 7). The $M_{\mathrm{w}}$ of the compound 7 was $686.49[\mathrm{M}+\mathrm{H}]^{+}$.

\section{Successful Generation of PTH-Lys ${ }^{27}$-AGC}

PTH-Lys ${ }^{27}$ peptide was reacted with NHS-AEEA-rGlu-C18 ester in alkaline solution to obtain PTH-Lys ${ }^{27}$-AGC. The chemical structure and high-performance LC (HPLC) curve of PTH-Lys ${ }^{27}$-AGC are shown in - Fig. 4. The results of mass spectrometry are shown in - Fig. $\mathbf{4 c}$; the molecular weight obtained was 4,744.02 Da, which is consistent with theoretical molecular weight of PTH-Lys ${ }^{27}$-AGC (4,744.15 Da). The modification site of PTH-Lys ${ }^{27}$-AGC was further investigated using peptide map analysis. PTH-Lys ${ }^{27}$-AGC was digested by trypsin and the peptide fragments are shown in - Fig. 5 . The digestion results in only five major peptide fragments which together account for the entire amino acid sequence of PTHLys $^{27}$-AGC ( - Table 2), and the molecular weight of the 27-34 segment $(1,570.84 \mathrm{Da})$ was consistent with the theoretical $M_{\mathrm{w}}$ of the sequence KLQDVHNF ( $\left.1,000.53 \mathrm{Da}\right)$ acylated by the -AEEA-rGlu-C18 side chain (570.31 Da). In addition, there is only one free amino group at position Lys $^{27}$ in this peptide segment. Therefore, we confirmed that the side chain AGC was successfully modified at Lys $^{27}$.

\section{Enhanced In Vitro Potency of PTH-Lys ${ }^{27}$-AGC}

The function of PTH-Lys ${ }^{27}$-AGC was assessed using in vitro activity on target cells. PTH acts via binding with the PTH1 receptor (PTH1R), a G protein-coupled receptor predominantly distributed in the bones and the kidneys. When PTH binds to its receptor, the cells release cyclic adenosine

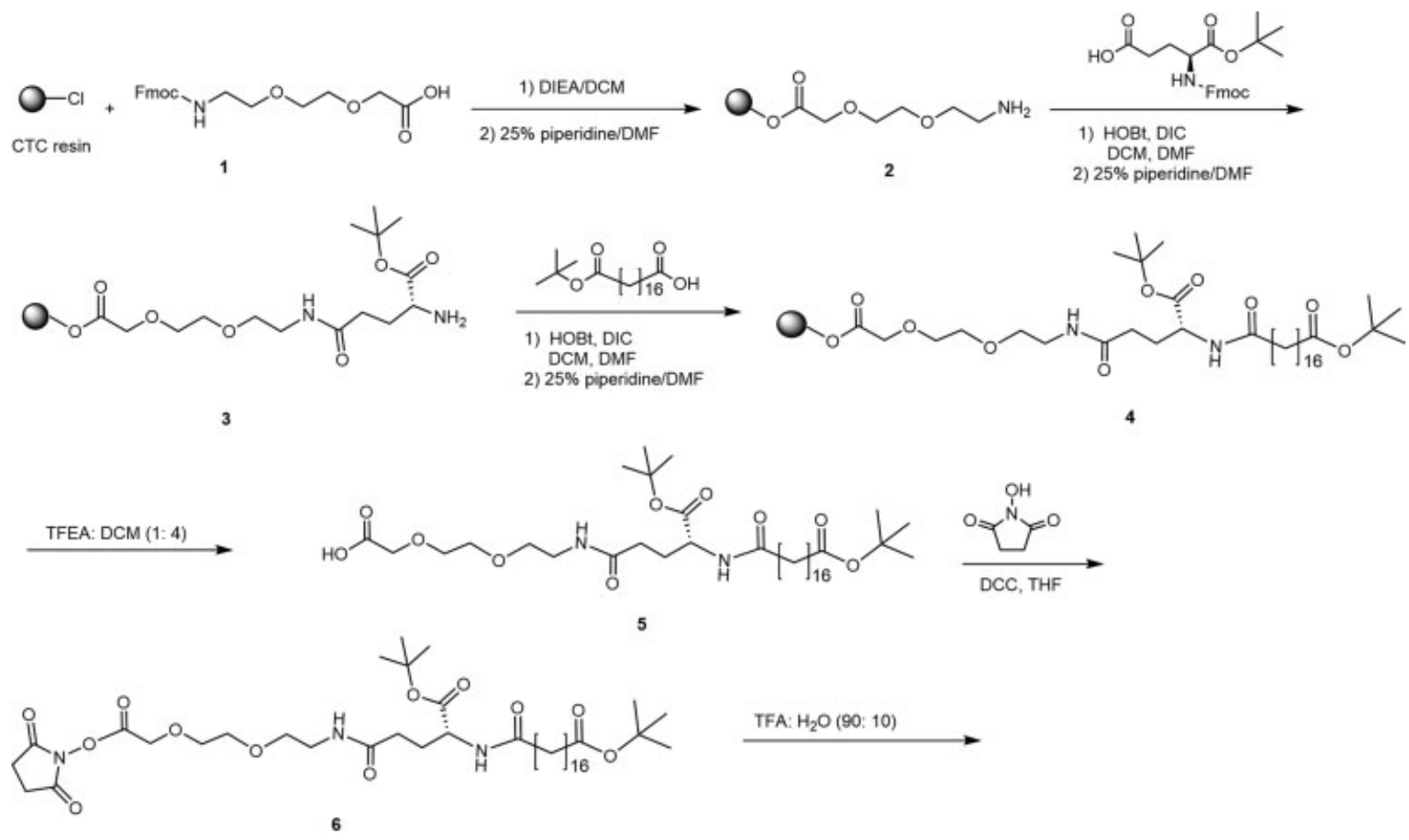<smiles>CC(CC(C)(C)C(=O)O)N[C@@H](CCC(=O)NCCOCCOCC(=O)ON1C(=O)CCC1=O)C(=O)O</smiles>

Fig. 3 Synthesis route of NHS-AEEA-yGlu-C18 ester (compound 7). 
A

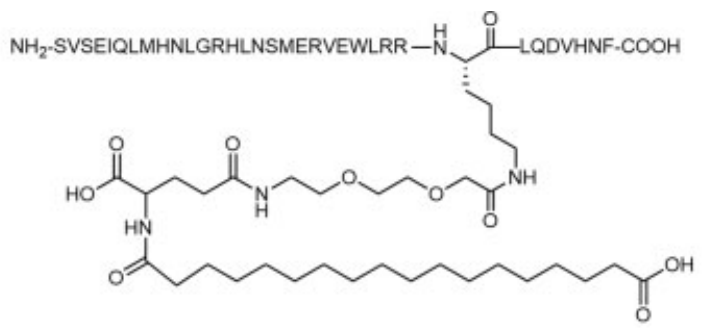

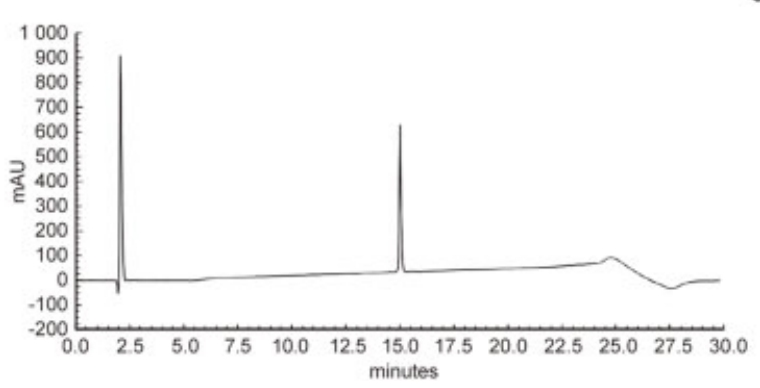

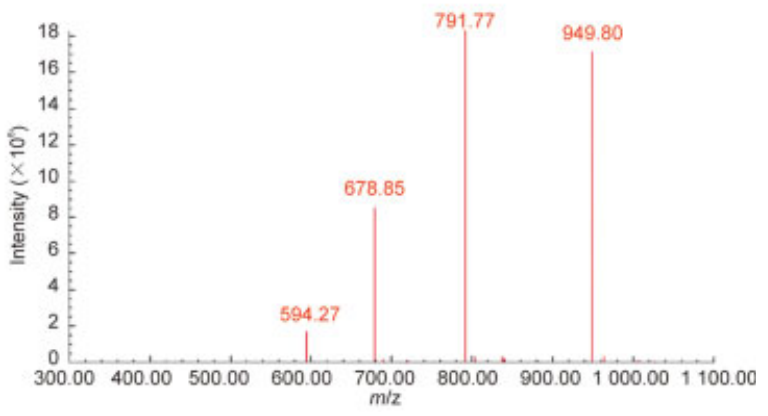

Fig. 4 (A) Chemical structure, (B) HPLC curve, and (C) mass spectrum of PTH-Lys ${ }^{27}$-AGC.

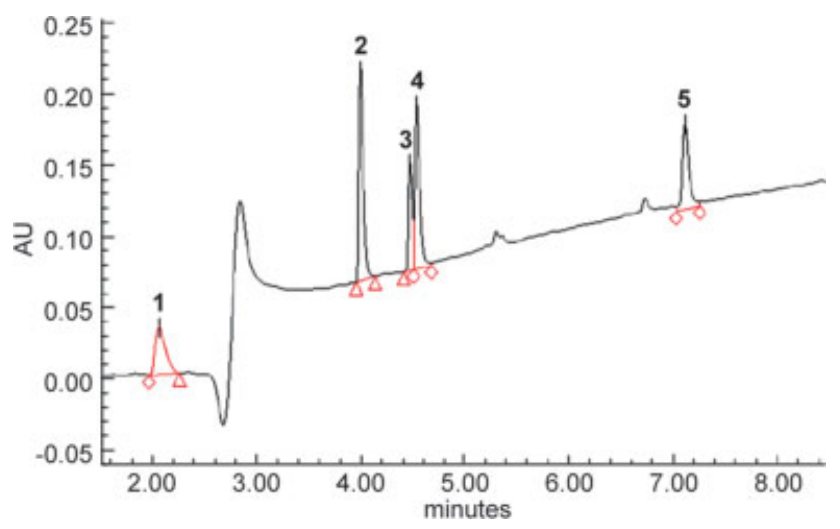

Fig. 5 Peptide map of PTH-Lys ${ }^{27-}$ AGC.

monophosphate (cAMP) to activate downstream pathways In this study, PTH-concentration-dependent cAMP formation in the cells was quantitatively analyzed using a timeresolved fluorescence resonance energy transfer (TR-FRET) immunoassay detection method. Cell response is defined as the TR-FRET signal.

As shown in - Fig. 6, a nonsigmoidal standard curve was observed, fitting to a weighted four-parameter logistic curve. $\mathrm{EC}_{50}$ values of PTH-Lys ${ }^{27}$-AGC and PTH1-34 were 84.98 and $196.75 \mathrm{pmol} / \mathrm{L}$, respectively, suggesting that the bioactivity of PTH-Lys ${ }^{27}$-AGC increased by approximately 2.3 -fold when compared with that of PTH1-34.

\section{PTH-Lys ${ }^{27}$-AGC Exhibited High HSA Affinity}

FA modifications can increase the half-life of peptides in vivo by facilitating albumin binding of FA segments. The main forces of albumin binding involve electrostatic and nonpolar interactions and the binding process is reversible. ${ }^{9}$ Several methods have been utilized to determine albumin affinity of FAs. Nonspecific adhesion and adsorption of FAs are regarded as the main obstacles in the establishment of effective in vitro
Table 2 Peptide map analysis of fragments and sequences of PTH-Lys ${ }^{27}$-AGC

\begin{tabular}{|l|l|l|}
\hline No. & $\mathbf{m} / \mathbf{z}$ & Peptide segment \\
\hline 1 & 886.51 & $14-20$ \\
\hline 2 & 858.61 & $21-26$ \\
\hline 3 & $1,483.78$ & $1-13$ \\
\hline 4 & 702.54 & $21-25$ \\
\hline 5 & $1,570.84$ & $27-34$ \\
\hline
\end{tabular}

methods. ${ }^{10}$ Blitz is a molecular interaction machine which evaluates binding capacity between the receptor and the ligand based on the principle of biolayer interferometry. A novel competitive receptor-binding assay with HSA was utilized in this study to examine albumin affinity by the Blitz system.

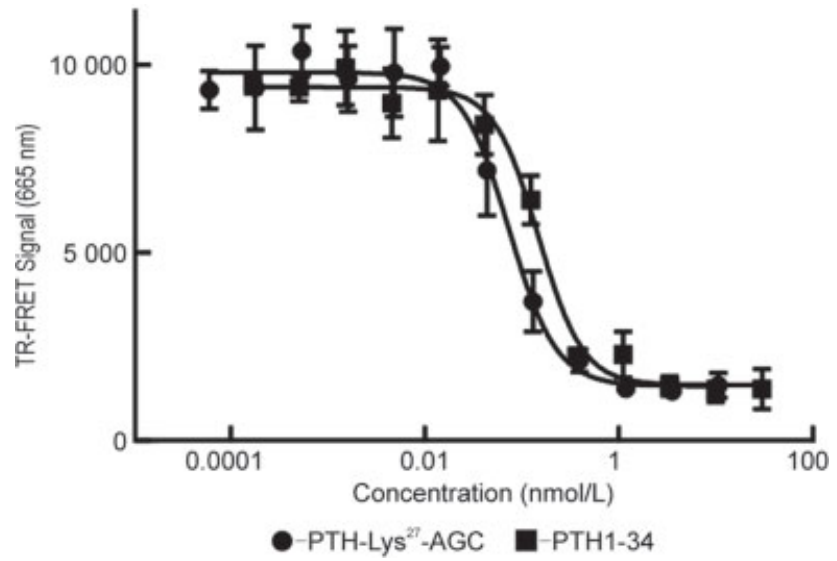

Fig. 6 In vitro bioactivity of PTH-Lys ${ }^{27}$-AGC and PTH1-34. 


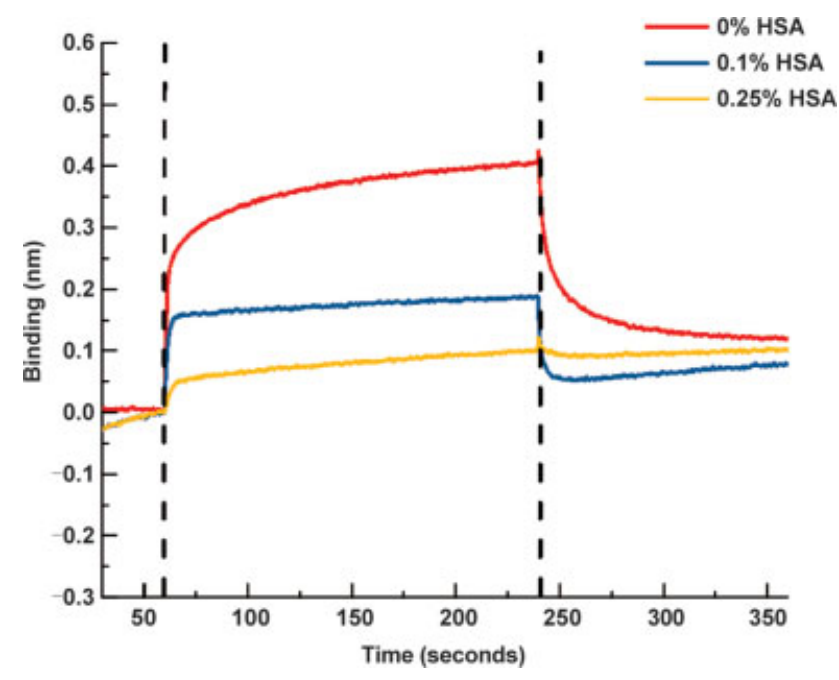

Fig. 7 PTH1R binding assay of PTH-Lys ${ }^{27}$-AGC in the presence or absence of albumin.

PTH-Lys ${ }^{27}$-AGC at 3,000 nmol/L was diluted in 0, 0.1, and $0.25 \%$ HSA buffer. - Fig. 7 shows that the binding signal of $0 \%$ HSA was at the highest when compared with 0.1 and $0.25 \%$ HSA, confirming the PTH receptor binding activities of PTHLys $^{27}$-AGC. However, with the increased concentration of HSA, the equilibrium signal of the samples gradually decreased, which may be attributed to the high albumin affinity of PTH-Lys ${ }^{27}$-AGC. HSA-bound peptides lost the ability to bind receptor and only free compound was absorbed by PTH1R. The albumin intervention reduced the amount of free drug in the system, inevitably leading to reduction in the signal. Our data thus suggest a strong albumin binding capacity of the newly generated compound.

Based on the fact that drugs absorbed by HSA cannot pass through the filter, an ultrafiltration method was further applied to prove whether there was a strong HSA affinity of PTH-Lys ${ }^{27}$-AGC. ${ }^{11,12}$ Our results showed that the addition of HSA has no effect on native PTH1-34 when compared with the control group, and the content of PTH1-34 in the filtrate was close to $99 \%$. However, with the addition of HSA, > $96 \%$ of PTH-Lys ${ }^{27}$-AGC was trapped, indicating the strong albumin binding affinity of PTH-Lys ${ }^{27}$-AGC after modification.

\section{Discussion}

FA-modified strategies have been the focus of research interest in prolonging half-life of peptide drugs. This strategy has been successfully applied to a variety of peptides, such as GLP-1, growth hormone, and calcitonin. ${ }^{13,14}$ In this study, we first reported the synthesis of a novel PTH1-34 analogue with FA modification.

The integral synthesis route of our FA-conjugated peptide is presented in this report. PTH1-34 contains three Lys residues as potential modification sites. In the current study, a novel PTH1-34 variant retaining a single Lys at position 27 was synthesized using recombinant protein expression. The side chain, -AEEA-rGlu-C18 diacid, was synthesized via a solid-phase reaction and linked to the PTH-Lys ${ }^{27}$ backbone through the free amino groups on lysine to produce the target compound (PTH-Lys ${ }^{27}$-AGC). Compared with the solid-phase and total chemical synthesis processes, the combined use of recombinant expression and solid-phase synthesis was recognized as having higher yield and a lower cost. In this synthesis route, a solid-liquid combined reaction strategy was adopted for modification of the peptide backbone. The one-step reaction in the liquid phase successfully avoids the formation of by-products and improves the purity of the target product.

Compared with PTH1-34, the newly generated PTHLys $^{27}$-AGC exhibited higher cellular bioactivity and strong HSA binding capacity, which may due to the presence of FA side chains. The side chain, -AEEA-rGlu-C18 diacid, contained bare carboxyl and nonpolar segments, which could bind HSA through electrostatic and nonpolar interactions, ${ }^{9}$ favoring the long acting of PTH-Lys ${ }^{27}$-AGC in therapeutic treatment. Encouragingly, once-weekly injection of PTH1-34 (Teribone) has been approved for osteoporosis therapy in Japan, despite the evidence that continuous PTH1-34 exposure reduced bone strength in mice in some literatures. ${ }^{15,16}$ Moreover, long-acting PTH1-34 analogs have been proved to maintain the serum calcium level of hypoparathyroidism patients. ${ }^{17,18}$ Therefore, long-acting PTH1-34 analogs have promising therapeutic value in the clinic.

\section{Experimental Section}

\section{Reagents and Equipment}

(2-[2-(9-Fluorenylmethoxycarbonyl) ethoxy] ethoxy) acetic acid (Fmoc-AEEA-OH; CS Bio-technology Co., Ltd.), N-(9fluorenylmethoxycarbonyl)-L-glutamic acid 1-tert-butyl ester (Fmoc-rGlu-OtBu; GL Biochemical Ltd.), 2-chlorotrityl chloride resin (2-CTC resin; GL Biochemical Ltd.), HOSu (GL Biochemical Ltd.), N,N'-dicyclohexylcarbodiimide (DCC; GL Biochemical Ltd.), 1-hydroxybenzotriazole (HOBt; GL Biochemical Ltd.), 18-(tert-butoxy)-18-oxooctadecanoic acid (C18 diacid; produced in our laboratory), and isopropyl thiogalactoside (IPTG; Shanghai Sango Biotech) were utilized. A recombinant E. coli DMR438-expressing PTH1-34 analog was generated in our laboratory. HSA was obtained from Sigma-Aldrich, the LANCE UltracAMP kit was purchased from PerkinElmer, and all other reagents were from Sinopharm Chemical Reagent Co., Ltd.

CSBio Peptide Synthesizer (CS Bio-technology Co., Ltd.), 5L Fermenter (Shanghai Baoxing Bio-technology Co., Ltd.), Ultimate 3000 HPLC (Thermo), Powerpac Basic Electrophoresis system (Bio-Rad), and ACQUITY Qda Mass detector (Waters) were employed for experiments.

\section{Medium Preparation}

To prepare the LB medium, $10.0 \mathrm{~g}$ tryptone, $5.0 \mathrm{~g}$ yeast extract, and $10.0 \mathrm{~g}$ sodium chloride were dissolved in $1 \mathrm{~L}$ of water. For the fermentation medium, $6.0 \mathrm{~g}$ glucose, $60.0 \mathrm{~g}$ glycerin, $15.0 \mathrm{~g}$ sodium chloride, $51.0 \mathrm{~g}$ disodium hydrogen phosphate, $12.0 \mathrm{~g}$ potassium dihydrogen phosphate, $15.0 \mathrm{~g}$ tryptone, $7.5 \mathrm{~g}$ ammonium citrate, $12.0 \mathrm{~g}$ ammonium sulfate, 
$1.5 \mathrm{~g}$ magnesium sulfate, $0.01 \mathrm{~g}$ calcium chloride, and $7.5 \mathrm{~g}$ yeast extract were dissolved into $3 \mathrm{~L}$ of water, and then adjusted $\mathrm{pH}=6.9$ using sodium hydroxide solution. Glycerin (75\%) containing $2 \%$ magnesium sulfate was utilized as the feeding medium.

\section{Recombinant Expression of PTH-Lys ${ }^{27}$}

PTH-Lys ${ }^{27}$-expressing recombinant E. coli DMR438 from the strain library were cultured in our laboratory. Inoculum was streaked onto plates and expanded in the LB medium, which was used to inoculate ( $1 \%$ inoculum) in a $3 \mathrm{~L}$ fermenter culture. The initial temperature was set as $37^{\circ} \mathrm{C}$ and the speed rotation was $500 \mathrm{rpm}$ at $\mathrm{pH}$ 6.9. When the level of dissolved oxygen started to increase rapidly, feeding was initiated at a speed of $25 \mathrm{~mL} / \mathrm{h}$. Within a suitable bacterial density range, $0.2 \mathrm{mmol} / \mathrm{L}$ IPTG was added to the fermentation medium. After 12 hours of incubation, the fermentation process was terminated.

Cell pellets were collected after centrifugation at $6,000 \mathrm{rpm}$ for 20 minutes, and then lysed using high-pressure homogenization. The lysed solution was recentrifuged to precipitate gp55-PTH inclusion bodies. To obtain target peptides, the gp55 fusion tag of inclusion bodies was removed via acid digestion and restriction enzyme digestion. Precipitated inclusion bodies were resuspended $(2 \%$, $\mathrm{w} / \mathrm{v}$ ) in water and heated to $80^{\circ} \mathrm{C}$, followed by addition of $6 \mathrm{~mol} / \mathrm{L}$ hydrochloric acid to a final concentration of 20 $\mathrm{mmol} / \mathrm{L}$. After a 1-hour incubation, acid digestion was terminated using $6.0 \mathrm{~mol} / \mathrm{L} \mathrm{NaOH}$ and $\mathrm{pH}$ adjusted to 8.30 . The suspension was centrifuged at 7,000 rpm for 20 minutes to remove the gp55 tag. The supernatant solution was collected for enzyme digestion. A specific amount of TEV protease was added into the solution with $10 \mathrm{U} / \mathrm{mg}$ substrate to release the target peptide sequence (PTH-Lys ${ }^{27}$ ). The reaction temperature was set as $30^{\circ} \mathrm{C}$ with gentle shaking. After a 2-hour incubation, the reaction was terminated by the addition of acetic acid.

The sample was subsequently purified via reverse-phase HPLC using a Kromasil C18 column $(10 \mathrm{~mm} \times 250 \mathrm{~mm}$, $10 \mu \mathrm{m})$ under the following conditions: mobile phase $\mathrm{A}$ ( $50 \mathrm{mmol} / \mathrm{L}$ phosphate buffer salt, $\mathrm{pH} 8.0$ ) and phase $\mathrm{B}$ (80\% acetonitrile $[\mathrm{ACN}]$ ) with gradient elution of 0 to 10 minutes: B 5\%; 10 to 15 minutes: B 5-40\%; 15 to 55 minutes: B 40-60\%. The column flow rate was 5 $\mathrm{mL} /$ minute and the detection wavelength was $280 \mathrm{~nm}$.

A LC-MS (Waters system) was used to determine the molecular weight of peptides. For LC, the mobile phase (composed of water $[\mathrm{A}]$ and $\mathrm{ACN}[\mathrm{B}]$, each containing formic acid $1 \%[\mathrm{v} / \mathrm{v}]$ ) was delivered at a rate of $0.3 \mathrm{~mL} \mathrm{~min}^{-1}$ using a gradient of 20 to $65 \%$ phase $B$ within 10 minutes at a detection wavelength of $214 \mathrm{~nm}$. Regarding MS parameters, electrospray interface conditions were set as follows: capillary voltage $0.8 \mathrm{kV}$, desolvation temperature $600^{\circ} \mathrm{C}$, source temperature $120^{\circ} \mathrm{C}$, and cone voltage $15 \mathrm{~V}$.

Synthesis and Activation of Side Chain (Compound 7) A solution of Fmoc-AEEA-OH (3 equiv., $635.3 \mathrm{mg}$ ) and DIEA ( 6 equiv., $501.6 \mu \mathrm{L}$ ) was added to the solid support ( $0.5 \mathrm{~g}$ of
2-CTC resin, $\mathrm{SD}=1.10 \mathrm{mmol} / \mathrm{g}$ ) to react with resin in the condensation reaction. The protecting Fmoc group was removed with $25 \%$ piperidine (in DMF, v/v). Fmoc-rGluOtBu (3 equiv., $702.1 \mathrm{mg}$ ), DIC (3 equiv., $265.3 \mu \mathrm{L}$ ), and HOBt (3 equiv., $222.9 \mathrm{mg}$ ) were dissolved into the mixture of DCM:DMF $(1: 1, v / v)$ to react with the bare amino group from AEEA. After completion of the reaction detected by ninhydrin, the protective Fmoc group was removed. C18 diacid (3 equiv., $608.8 \mathrm{mg}$ ) was subsequently added for condensation and stirred at room temperature for 1 hour. Next, trifluoroethanol (20\% in DCM, v/v) was used to cleave the side chain from the resin. Rotary evaporation was performed to remove organic solvents to obtain compound $\mathbf{5}$ with the tertiary butyl-protecting carboxyl group.

Compound 5 was activated with HOSu to form NHS ester. DCC (1.5 equiv., $169.9 \mathrm{mg}$ ) and HOSu (1.5 equiv., $94.9 \mathrm{mg}$ ) were dissolved in tetrahydrofuran. HOSu reacted with the terminal carboxyl group of the side chain under the action of the condensing agent (DCC). The insoluble by-product was filtered and the organic solvent evaporated to obtain compound 6 , which was redissolved in $90 \%$ trifluoroacetate (in water) to remove tert-butyl protection to get side chains with NHS esters (compound 7). At last, cold diethyl ether was added to precipitate compound $\mathbf{7}$, and precipitation was collected by centrifugation at $10000 \mathrm{rpm}$ for 10 minutes. After drying, compound 7 was dissolved in ACN with the final concentration of $30 \mathrm{mg} / \mathrm{mL}$.

\section{Modification of PTH-Lys ${ }^{27}$ Using Side Chains}

PTH-Lys ${ }^{27}$ peptide ( $100 \mathrm{mg}$ ) was dissolved in $0.1 \mathrm{~mol} / \mathrm{L}$ triethylamine solution, and then the side-chain NHS-AEEArGlu-C18 diacid (compound 7) was added dropwise. The reaction was monitored using the HPLC method, and terminated with $10 \%$ acetic acid solution. Mobile phases of A and B were $0.1 \%$ trifluoroacetic acid (TFA) in water and $0.1 \%$ TFA in $\mathrm{ACN}$, respectively. The detection wavelength was $215 \mathrm{~nm}$, and the column temperature was $40^{\circ} \mathrm{C}$. The elution gradient was from 20 to $80 \%$ over 16 minutes.

prep-HPLC (Waters) with a C18 reversed-phase silica gel column was used to purify the final product. The mobile phase was set as the following: buffer $A(0.1 \%$ TFA [v/v] water $)$ and buffer $B(0.1 \%$ TFA $[v / v]$ ACN). The wavelength was set as $280 \mathrm{~nm}$. Target compounds were obtained using gradient elution from 20 to $60 \%$ phase B. Samples with $>95 \%$ purity were collected and underwent rotary evaporation to remove organic solutions. The final product was achieved after lyophilization.

\section{In Vitro Cell Bioactivity of PTH-Lys ${ }^{27}$-AGC}

First, $5 \mu \mathrm{L}$ of cell suspension was added to a 384-well plate (3,000 cells/well). $5 \mu \mathrm{L}$ PTH1-34 or PTH-Lys ${ }^{27}$-AGC solution ( $30 \mathrm{nmol} / \mathrm{L}$ to $0.15 \mathrm{pmol} / \mathrm{L}$ ) was added to the cell suspensions and incubated at room temperature. After 30 minutes, the cells were lysed using cell lysis solution to release intracellular CAMP. Mixed solutions of ULight-anti-cAMP and europium-labeled cAMP $(10 \mu \mathrm{L})$ were injected for quantification of cAMP levels. 
Experimental data were analyzed with GraphPad Prism5 software. The logarithm of the sample concentration was set as the $X$-axis and the corresponding fluorescence signal as the $Y$-axis. A four-parameter equation was selected for fitting.

\section{In Vitro HSA-Binding Test}

Blitz (PALL Corporation) is a molecular interaction apparatus based on the principle of biolayer interferometry. Here, biotinylated PTH1R was incubated on a streptomycin-coated biosensor. PBST (pH 7.4 containing $0.2 \%$ Tween 20 ) was used as an equilibration solution. PTH-Lys ${ }^{27}$-AGC was diluted to a final concentration of $3,000 \mathrm{nmol} / \mathrm{L}$ using $0,0.1$, and $0.25 \% \mathrm{HSA}$ buffer. Next, after a 60 -second baseline step, biosensors were dipped into wells containing PTH-Lys ${ }^{27}$-AGC for 180 seconds during the association phase, followed by the dissociation phase of 120 seconds in the corresponding running buffer. Data were analyzed using Blitz PRO1.2 software.

Another in vitro HSA-binding test based on ultrafiltration was utilized. PTH1-34 or PTH-Lys ${ }^{27}$-AAPC was dissolved in PBS 7.4 solution containing $0.5 \mathrm{mg} / \mathrm{mL}$ HSA (test group) and $0 \mathrm{mg} / \mathrm{mL}$ HSA (control group) with the final concentration of $0.1 \mathrm{mg} / \mathrm{mL}$. Sample solution was added into an ultrafiltration tube (30 kDa, Millipore) and centrifuged at 12,000 rpm for 10 minutes. The peptide concentration of filtrate was determined by HPLC.

\section{Conflict of Interest}

None.

\section{References}

1 Klibanski A, Adams-Campbell L, Bassford T, et al; NIH Consensus Development Panel on Osteoporosis Prevention, Diagnosis, and Therapy. Osteoporosis prevention, diagnosis, and therapy. JAMA 2001;285(06):785-795

2 Dempster DW, Cosman F, Parisien M, Shen V, Lindsay R. Anabolic actions of parathyroid hormone on bone. Endocr Rev 1993;14 (06):690-709

3 Ramírez-Andersen HS, Behrens C, Buchardt J, et al. Long-acting human growth hormone analogue by noncovalent albumin binding. Bioconjug Chem 2018;29(09):3129-3143
4 Zorzi A, Linciano S, Angelini A. Non-covalent albumin-binding ligands for extending the circulating half-life of small biotherapeutics. Med Chem Comm 2019;10(07):1068-1081

5 van der Vusse GJ. Albumin as fatty acid transporter. Drug Metab Pharmacokinet 2009;24(04):300-307

6 Menacho-Melgar R, Decker JS, Hennigan JN, Lynch MD. A review of lipidation in the development of advanced protein and peptide therapeutics. J Control Release 2019;295:1-12

7 Bech EM, Pedersen SL, Jensen KJ. Chemical strategies for half-life extension of biopharmaceuticals: lipidation and its alternatives. ACS Med Chem Lett 2018;9(07):577-580

8 Li XW, Wu Y, Huang ZQ et al. Recombinant expression of plecanatide in Escherichia coli. [in Chinese] Chinese Journal of Pharmaceuticals 2020;51(10):1282-1287

9 Fujiwara S, Amisaki T. Fatty acid binding to serum albumin: molecular simulation approaches. Biochim Biophys Acta 2013; 1830(12):5427-5434

10 Lau J, Bloch P, Schäffer L, et al. Discovery of the once-weekly glucagon-like peptide-1 (GLP-1) analogue semaglutide. J Med Chem 2015;58(18):7370-7380

11 Barré J, Chamouard JM, Houin G, Tillement JP. Equilibrium dialysis, ultrafiltration, and ultracentrifugation compared for determining the plasma-protein-binding characteristics of valproic acid. Clin Chem 1985;31(01):60-64

12 Marner L, Ettrup A, Haahr M, et al. Comparison of ultrafiltration and equilibrium dialysis for estimation of protein binding. Neuroimage 2010;52:S200

13 Madsen K, Knudsen LB, Agersoe H, et al. Structure-activity and protraction relationship of long-acting glucagon-like peptide-1 derivatives: importance of fatty acid length, polarity, and bulkiness. J Med Chem 2007;50(24):6126-6132

14 Lau J, Hansen TK, Madsen K, et al. Novel GLP-1 derivatives: WO Patent 2005027978. March, 2005

15 Tay D, Cremers S, Bilezikian JP. Optimal dosing and delivery of parathyroid hormone and its analogues for osteoporosis and hypoparathyroidism - translating the pharmacology. Br J Clin Pharmacol 2018;84(02):252-267

16 Choudhary S, Santone E, Yee SP, et al. Continuous PTH in male mice causes bone loss because it induces serum amyloid $\mathrm{A}$. Endocrinology 2018;159(07):2759-2776

17 Guo J, Khatri A, Maeda A, Potts JT Jr, Jüppner H, Gardella TJ. Prolonged pharmacokinetic and pharmacodynamic actions of a pegylated parathyroid hormone (1-34) peptide fragment. J Bone Miner Res 2017;32(01):86-98

18 Krishnan V, Ma YL, Chen CZ, et al. Repurposing a novel parathyroid hormone analogue to treat hypoparathyroidism. Br J Pharmacol 2018;175(02):262-271 